Barbara, personal communication). A report from New York found that six of 221 patients with cancer who had undergone multiple transfusion had antibodies to HTLV-I, but this high rate has not yet been confirmed elsewhere. ${ }^{20}$ The cost-benefit analysis for screening donors must include the low prevalence of HTLV-I in Britain, the relatively high incidence of false positive reactions, and the low risk of disease developing after infection. Despite these problems the American Red Cross has already begun screening blood donations in the United States for antibodies against HTLV-I, probably in view of potential litigation in the future.

Investigation of HTLV-I infection in clinical practice needs to be extended in Britain; it should no longer be confined only to patients from endemic areas as sporadic cases have been reported in low risk populations from most European countries. ${ }^{21}$ Studies on HTLV-I in Britain to date have been valuable but unplanned and uncoordinated. The Public Health Laboratory Service Communicable Disease Surveillance Centre seems the appropriate organisation to define the epidemiological features of HTLV-I in Britain, and its acceptance of the task seems long overdue.

JONATHAN WEBER

Senior Lecturer in Infectious Diseases,

Department of Medicine,

Royal Postgraduate Medical School,

London W12 0HS
1 Weiss RA. Human T-cell leukaemia/lymphoma virus. In: Weiss R, Coffin J, Varmus H, Teich N eds. RNA tumour viruses. New York: Cold Spring Harbour, 1984:1244-2.

Hinuma Y, Komoda H, Chosa T, et al. Antibody to adult T-cell leukaemia virus associated antigen (ATLA) in sera from patients with ATL and controls in Japan. Int $f$ Cancer 1982;29:631-5.

Gessain A, Barin F, Vernant J, et al. Antibodies to HTLV-I in patients with tropical spastic paraparesis. Lancet 1985; ;i: 1247 .

Murphey EL, Hanchard B, Figueroa JP, et al. Modelling the risk of ATLL in persons infected with HTLV-I. Int 7 Cancer 1989:43:250-3.

5 Roman GC, Osame M. Identity of HTLV-I associated TSP and myelopathy. Lancet 1988;i:65 I

6 Morgan O, Rodgers-Johnson P, Mora C, Char G. HTLV-I and polymyositis in Jamaica. Lancet 1989;ii:1184-6.

Tachibana N, Okayama A, Ishizaki J, et al. Suppression of tuberculin skin reactivity in healthy HTLV-I carriers in Japan. Int $f$ Cancer 1988;42:829-31.

8 Levine PH, Blattner W, Clark J, et al. Geographic distribution of HTLV-I and identification of a new high-risk population. Int 7 Cancer 1988;42:7-12.

9 Tajima K, Kamura S, Ito S, et al. Epidemiological features of HTLV-I carriers and incidence of ATL in an ATL-endemic island. Int 7 Cancer 1987;40:741-6.

10 Tedder RS, Shanson DC, Jeffries DJ, et al. Low prevalence in the UK of HTLV-I and HTLV-III infection in subjects with AIDS, lymphadenopathy syndrome and at risk of AIDS. Lancet 1984;ii:125-8.

11 Robert-Guroff $M$, Weiss S, Giron J, et al. Prevalence of antibodies to HTLV-1, 2 and 3 in IV drug users from an AIDS endemic area. $\mathcal{F} A M A$ 1986;255:3133-7.

12 Okochi K, Sato H, Hinuma Y. A retrospective study on transmission of ATL virus by blood transfusion: seroconversion in recipients. Vox Sang 1984;46:245-53.

13 Tajima K, Tominaga S, Suchi T, et al. Epidemiological analysis of the distribution of antibody to ATLV: possible horizontal transmission. Fapanese foumal of Cancer Research 1982;73:893-901. 4 Komuro A, Hayami M, Fujii H, et al. Vertical transmission of ATL virus. Lancet 1983;i:240.

15 Ando Y, Saito K, Nakano S, et al. Bottle feeding can prevent transmission of HTLV-I from mothers to their babies. F Infect 1989;19:25-9.

16 Hirose S, Kotani S, Uemura Y, et al. Milk borne transmission of HTLV-I in rabbits. Virology 1988;162:487-9.

17 Cruickshank JK, Richardson JH, Morgan OStC, et al. Screening for prolonged incubation of HTLV-I infection in British and Jamaican relatives of British patients with tropical spastic paraparesis. Br Med f 1990:300:300-4

18 Weber JN, Banatvala N, Clayden S, et al. HTLV-I infection in Papua New Guinea: evidence for serologic false positivity. $\mathcal{F}$ Infect Dis 1989;159:1025-8.

19 Williams AE, Fang CT, Slamon D, et al. Seroprevalence and epidemiologic correlates of HTLV-I infection in US blood donors. Science 1988;240:643-6.

20 Minamoto G, Gold J, Scheinberg D, et al. Infection with HTLV-I in patients with leukaemia. $N$ Engl f Med 1988;318:219-22.

21 Wyld J, Tosswill J, Mortimer P, Weber JN. Sporadic HTLV-I in the UK. Lancet (in press).

\title{
Fixed dose procedures for tests on animals
}

\section{Agreement has been reached on the end of the $L D_{50}$}

The $\mathrm{LD}_{50}$ test is a procedure to determine the dose of a chemical that kills half of the animals exposed. The test has long been criticised as simplistic in using death as the sole end point, rather than a screen of biological responses. It has come under increasing challenge, too, by critics of experiments on animals, who claim that it is contrary to the humanity of animal protection legislation in the United Kingdom (Animals (Scientific Procedures) Act 1986) and the European Economic Community (Directive 86/609). Yet the $\mathrm{LD}_{50}$ has remained an important part of the data required to classify the hazards of substances ranging from household products to medicines - for example, in the European Community under Directive $79 / 831$, where the laboratory method is fully described in annex V. A similar, universally accepted procedure is described in the Organisation for Economic Cooperation and Development test guideline 401.

In 1984 the British Toxicology Society took the initiative, with the support of the Health and Safety Executive, to develop and prove an alternative, more humane technique to replace the oral $\mathrm{LD}_{50}$ test. The new "fixed dose procedure" was designed to use fewer animals than the $\mathrm{LD}_{50}$ test; not to require death as the end point; to cause less pain and distress; to permit the hazard classification of compounds on the basis of acute toxicity; and to provide better information about toxic effects that is needed for human risk assessment.

The basis of the new procedure is that a few rodents (usually only three) are given the test material by using one of a series of fixed doses related to the agreed limits in the accepted classification system. A dose is selected to cause "evident" toxicity but not death. If there is no prior knowledge to guide selection then the lowest dose level is chosen first. All of the animals are closcly observed, and any that show signs of severe effects are killed humanely. If the first dose has no effect then the next higher level is used according to the classification scheme.

The feasibility of this fixed dose procedure was proved in an initial interlaboratory validation trial in Britain, which was supported by the Health and Safety Executive.' The results were promising enough for a major collaborative study to be arranged in 33 laboratories in 11 countries with the support of the European Commission and in Britain with the support of the Department of Health, the Home Office, and the Health and Safety Executive.

The results and those of possible alternative methods were presented last year at a seminar in Brussels that was attended by official representatives and scientists from the states of the European Community and from many other countries. The new fixed dose procedure was generally well received as a practical and efficient method of classifying the toxic hazards of chemicals for regulatory purposes. It gained support even from countries that use different reference limits or different categories.

At the end of the meeting the European Commission, through Directorate General XI, formally stated that an initiative to promote the rationalisation of classification schemes based on acute toxicity data would be fully supported by the European Community. Moreover, formal discussions to gain general acceptance of the new method would be arranged within the community, with the Organisation for Economic Cooperation and Development, and with other international organisations.

This represents a major advance in the difficult borderland between legal and regulatory requirements, scientific procedures, and considerations of animal and human welfare. It has 
come from hard work by people in the British Toxicology Society and in industrial laboratories to validate the fixed dose procedure, from the support of official bodies, and from some encouragement (and some disparagement) by animal welfare groups. The important general conclusion seems to be that changes in animal experimentation are most likely to be achieved on the basis of clear evidence of utility and when all concerned understand the purposes and limitations of the alternative procedure.

A D DAYAN

Professor of Toxicology, St Bartholomew's Hospital,

London EC1 7ED

1 Van den Heuvel MJ, Dayan AD, Shillaker RO. Evaluation of the BTS approach to the testing of substances and preparations for their acute toxicity. Hum Toxicol 1987;6:279-91.

\section{Leukaemia and cancer chemotherapy}

\section{The risk is acceptably small but may be reducible further}

In the past 15 years cytotoxic chemotherapy has revolutionised the prognosis for patients with some kinds of tumours. Because of this clinicians are now paying far more attention to the long term risks of such treatment, including leukaemia, sterility, cardiopulmonary disease, and altered immunity. ${ }^{1-3}$ Reports have compared the incidence of leukaemia in patients treated for various cancers with that in healthy populations and have examined the actuarial incidence of leukaemia in subgroups receiving different treatments. ${ }^{+5}$ This approach, however, does not overcome one source of potential biasthat, for instance, Hodgkin's disease itself or certain stages of the disease may be associated with an increased risk of leukaemia.

More recently, Kaldor et al reported two studies-one in Hodgkin's disease ${ }^{6}$ and the other in ovarian cancer ${ }^{7}$ - that overcame this problem by using a case-control design within large populations of patients with these two tumours. They found 114 cases of leukaemia in 99113 survivors of ovarian cancer and matched each of these with three (four in the case of 35283 German patients) patients without leukaemia. ${ }^{8}$ For Hodgkin's disease they found 163 cases of leukaemia among 29552 patients, and these were each matched with three controls with Hodgkin's disease without leukaemia.

In the study of ovarian cancer, chemotherapy alone was associated with a 12 -fold increase in relative risk compared with surgery alone. Patients receiving chemotherapy and radiotherapy had a relative risk of $10 \cdot 0$; radiotherapy alone did not increase the risk above that of surgery. All alkylating agents clearly increased the risk of leukaemia, as did the combination of doxorubicin and cisplatin.

For Hodgkin's disease chemotherapy alone was associated with a relative risk of leukaemia of 9.0 as compared with radiotherapy alone. Patients treated with both modalities did not have a further increase in risk, though treatment with more than six cycles of nitrogen mustard and procarbazine increased the relative risk to $14 \cdot 0$. Radiotherapy caused a dose related increase in risk. When adjustment was made for the chemotherapy used there was a twofold increase in risk of leukaemia in those who had had a splenectomy.

In general these reports confirm the results of previous cohort studies, ${ }^{+5}$ but they do not provide a precise estimate of the risk of leukaemia as there was appreciable heterogeneity of the rates of leukaemia depending on the source of the data. Most data were from population based cancer registries, but some were from single institutions. The crude incidence of leukaemia was eight times higher in single hospitals than in registries for ovarian carcinoma; the comparable figure for Hodgkin's disease showed a near fourfold difference. These data might possibly reflect patient selection and more intensive treatment in single institutions, but an alternative explanation is that there was underrecognition of a second malignancy in patients from tumour registries. Supporting this hypothesis is the finding that nine of 11 cases of myelodysplastic syndrome in patients with ovarian cancer occurred in one institution that accounted for $4 \%$ of the patients.

What, then, is the risk that chemotherapy will induce leukaemia, and does cost-benefit analysis suggest that such chemotherapy should continue to be used? As a corollary to this, can the therapeutic efficacy of chemotherapy be maintained while its toxicity is reduced? Data from multiple studies in ovarian cancer suggest that the risk of leukaemia is four to five times that in a normal population. The risk is almost entirely related to exposure to alkylating agents, though Kaldor et al also implicate the combination of cisplatin and doxorubicin, ${ }^{7}$ a finding recently reported by others. ${ }^{9}$ The risk rises with total dose used. ${ }^{37}$ For patients with Hodgkin's disease given chemotherapy the risk in Kaldor's study was a little over five times that in those receiving radiotherapy alone. ${ }^{6}$ The actuarial risk of leukaemia five to 10 years after treatment for Hodgkin's disease is $2-10 \%,{ }^{1011}$ and a second solid malignancy has been increasingly reported. ${ }^{+5}$

Cost-benefit analysis in advanced Hodgkin's disease clearly favours chemotherapy: the risk of drug induced leukaemia is relatively small, and such treatment greatly increases the chances of long term survival. Before the introduction of effective chemotherapy survival in patients with advanced disease was less than $5 \%$ at five years - nowadays over half of such patients survive long term. ${ }^{12}$ The data of Kaldor et al may, however, provide a further argument for the trend away from splenectomy, and they certainly argue against unnecessarily prolonged chemotherapy and for the move towards regimens that result in less exposure to alkylating agents and so in lower rates of leukaemia and sterility. ${ }^{13}$

The results are less clear for patients with advanced ovarian carcinoma, in whom prolonged survival after chemotherapy is uncommon. No single randomised study has been large enough to identify the most effective type of chemotherapy, though cisplatin combinations are commonly recommended. A meta-analysis of over 50 randomised studies is in progress and should provide clearer guidance. ${ }^{1+}$ The risk of leukaemia remains small, and the data suggest that there is a clear benefit for some form of chemotherapy, but as prolonged treatment with alkylating agents has been shown to result in an increasing risk of leukaemia such treatment should be avoided when possible.

The potential for chromosomal damage and the development of myelodysplatic syndrome have been recognised as risks of chemotherapy for some years. ${ }^{15-18}$ Chromosomal changes characteristic of leukaemia have been identified in patients treated with cytotoxic agents, ${ }^{19}$ and these include damage to genes concerned with haemopoietic regulation. ${ }^{20}$ 